\title{
Fuel Saving Strategies for Separated Spacecraft Interferometry
}

Timothy McLain

Mechanical Engineering Department, Brigham Young University, mclain@byu.edu

Randal W. Beard

Department of Electrical and Computer Engineering, Brigham Young University, beard@ee.byu.edu

Christopher A. Bailey

Flight Systems Engineering Department, Hernandez Engineering

Follow this and additional works at: https://scholarsarchive.byu.edu/facpub

Part of the Mechanical Engineering Commons

\section{Original Publication Citation}

Bailey, C., McLain, T., and Beard, R. Fuel Saving Strategies for Separated Spacecraft Interferometry, Proceedings of the AIAA Guidance, Navigation, and Control Conference, AIAA-2000-4441, August 2000, Denver, Colorado.

\section{BYU ScholarsArchive Citation}

McLain, Timothy; Beard, Randal W.; and Bailey, Christopher A., "Fuel Saving Strategies for Separated Spacecraft Interferometry" (2000). Faculty Publications. 1920.

https://scholarsarchive. byu.edu/facpub/1920

This Conference Paper is brought to you for free and open access by BYU ScholarsArchive. It has been accepted for inclusion in Faculty Publications by an authorized administrator of BYU ScholarsArchive. For more information, please contact ellen_amatangelo@byu.edu. 


\title{
FUEL SAVING STRATEGIES FOR SEPARATED SPACECRAFT INTERFEROMETRY
}

\author{
Christopher A. Bailey ${ }^{1} \quad$ Timothy W. McLain ${ }^{2 *} \quad$ Randal W. Beard ${ }^{3}$ \\ ${ }^{1}$ Flight Systems Engineering Department, Hernandez Engineering, Houston, Texas 77058 \\ ${ }^{2}$ Department of Mechanical Engineering, Brigham Young University, Provo, Utah 84602 \\ ${ }^{3}$ Department of Electrical and Computer Engineering, Brigham Young University, Provo, Utah 84602
}

\begin{abstract}
Separated spacecraft interferometry missions will require spacecraft to move in a coordinated fashion to ensure minimal and balanced consumption of fuel. This paper develops strategies for determining interferometry mission plans that result in significant fuel savings over standard approaches. Simulation results demonstrate that valuable reductions in fuel consumption can be realized by combining the retargeting and imaging maneuvers required to image multiple stellar sources. Fuel-optimal imaging strategies have been developed for two-spacecraft interferometry missions similar to the proposed Space Technology 3 mission using chained local optimization methods. Based on these strategies, sampling pattern guidelines for space-borne interferometry missions have been developed.
\end{abstract}

\section{INTRODUCTION}

Space-based optical interferometry has been identified by NASA as one of the key technologies in furthering the scientific exploration of the universe in the next century. NASA's Origins Program will use the fundamentals of interferometry to form a sophisticated space-based telescope as the primary tool for future space missions to image stars and distant planetary systems with new levels of accuracy. This paper develops fuel saving schemes for separated spacecraft stellar interferometry missions. The objective is to minimize the amount of propellant mass required to perform the mission objectives while ensuring that no spacecraft in the formation is left with insufficient fuel to complete the mission.

Interferometers image stellar targets in a significantly different way than conventional telescopes, which use a single large primary mirror. An optical

\footnotetext{
* Corresponding author, email: tmelaincet.byu.edu
}

interferometer samples parallel wavefronts at two or more locations and combines the light to produce an interference pattern. From these interference patterns or fringes, high angular resolution images of the source can be constructed. Optical interferometry requires a precisely controlled separation between the light collecting apertures. The resolution of the interferometer is proportional to the largest baseline of separation between apertures.

Shao and Colavita ${ }^{1}$ give a comprehensive technical review of ground-based interferometry and the advantages of space-based interferometry. Joshi ${ }^{2}$ provides an excellent introduction to interferometry and offers a technical discussion on how images are constructed from interferometric instrument interference patterns. Figure 1 shows an example of the relationship between the source and observation plane. The spacecraft collect observations in the form of interference fringes from the source at locations given by $\left(\eta_{1}, \nu_{1}\right)$ and $\left(\eta_{2}, \nu_{2}\right)$ in the observation plane. Joshi explains that the van Cittert-Zernike

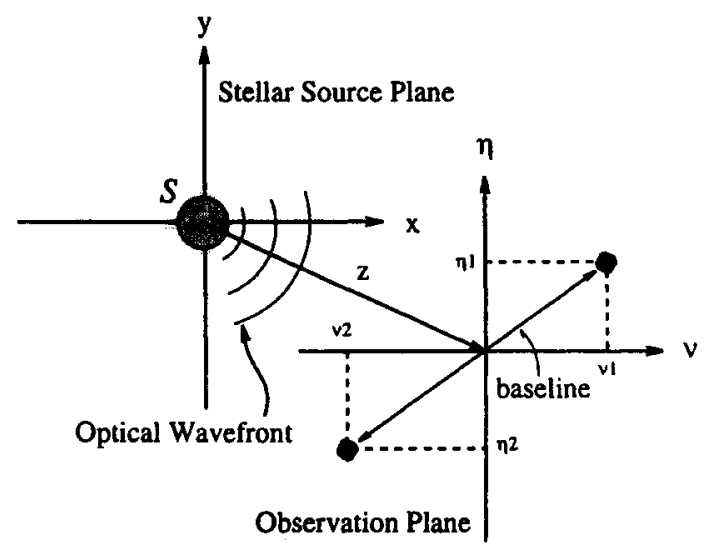

Figure 1: Synthetic aperture imaging scenario 
result is a two-dimensional Fourier transform and is the basis for interferometric imaging. An irradiance pattern $I(x, y)$, that forms the image of the source $S$ can be constructed from this transform by

$$
I(x, y) \triangleq \int_{-\infty}^{\infty} \int_{-\infty}^{\infty} \mu(u, v) e^{i 2 \pi(u x+v y)} d u d v
$$

$\mu(u, v)$ is called the complex mutual coherence function. Points in the $(u, v)$ plane are mapped from locations of the spacecraft in the $(\nu, \eta)$ plane by

$$
\begin{gathered}
u \triangleq \frac{\eta_{1}-\eta_{2}}{z \lambda} \\
v \triangleq \frac{\nu_{1}-\nu_{2}}{z \lambda}
\end{gathered}
$$

where $\lambda$ is the wavelength of emitted light and $z$ the distance to the source. The mutual coherence function is calculated from amplitude and phase measurements made in the observation plane $(\nu, \eta)$ for each point in the $(u, v)$ plane. Due to the symmetry properties of Fourier transforms, celestial targets that are spherical (e.g., a single star) only require the sampling of a radial line in the $(u, v)$ plane. These targets are called 1D targets. Alternatively, nonspherical targets (e.g., a binary star) require sampling of half of the $(u, v)$ plane, and are termed $2 \mathrm{D}$ targets.

Separated spacecraft formations offer an intriguing platform for space-based interferometry. As illustrated in Figure 2, interferometry requires three basic formation maneuvers. The first is retargeting.

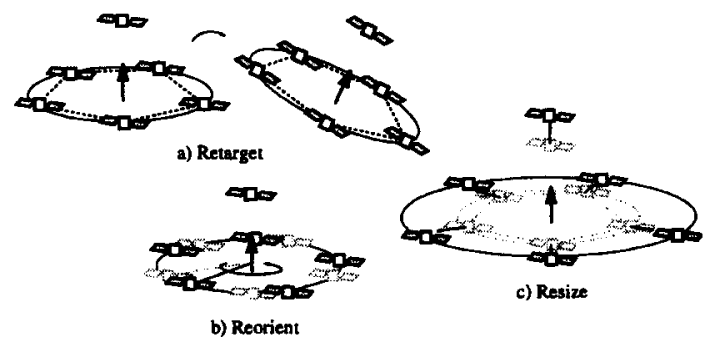

Figure 2: Repositioning maneuvers for space-based optical interferometry.

This involves realigning the light collecting apertures with a new celestial target. To do so, the spacecraft are rotated about some point in space to redirect the optical bore sight of the interferometer at the new source. The next is reorienting the formation about the bore sight of the interferometer. This provides a degree of freedom used for sampling points in the $(u, v)$ plane at a constant baseline separation between spacecraft. The last is resizing, where the formation maintains the same observation direction while spacecraft move to change the baseline separation. A typical mission will involve a combination of these maneuvers in order to sample the $(u, v)$ plane for a collection of stars.

Retarget, reorient, and resize maneuvers all require active thrusting and therefore expend onboard propellant, making fuel the major limitation to the length of the mission. It is therefore imperative that formation maneuvers be performed in a way that minimizes the expended fuel. An additional complication however, is that the useful lifetime of the mission is limited by the first spacecraft to run out of fuel. It may be that in performing fuel minimal retarget maneuvers, one of the spacecraft will burn fuel at a significantly faster rate than the others. ${ }^{3,4}$ An additional goal is therefore to perform formation maneuvers such that the fuel on each spacecraft is equalized.

For individual retarget, resize, and reorient maneuvers, it is possible to derive fuel minimizing/equalizing maneuvers. The general fuel minimization/equalization problem has been solved for individual retarget and reorientation maneuvers in Ref. 3 for constrained (i.e., sensor-lock) maneuvers, and in Ref. 4 for unconstrained maneuvers. In Ref. 5 a solution to the fuel minimization/equalization problem for resize maneuvers in the context of the planned ST3 mission is given.

Equalizing or minimizing the fuel for individual maneuvers may be thought of as a local fuel optimization problem. In this paper we are primarily concerned with the global fuel optimization problem, i.e., given that each individual formation maneuver can be performed in a fuel minimizing/equalizing manner, how should the maneuvers be sequenced to minimize/equalize the overall fuel usage?

There are two reasonable alternatives: The first and simpler alternative is to find the fuel optimal sequence of retarget maneuvers, assuming that once the formation has been retargeted to a single star, that all $(u, v)$ points will be sampled for that star (through a sequence of reorient and resize maneuvers), before retargeting to the next star. This approach, which we call the targeting maneuver optimization (TMO) strategy, is discussed in detail in Ref. 5

If the angular separation between two stars is small, then considerable fuel saving can be obtained by mixing $(u, v)$ points from one star with those of another. The second alternative, which is the focus of this paper, is to solve the fuel optimization problem allowing arbitrary mixing between reorient, resize, and retarget maneuvers. In other words, from a fuel point of view it may be better to collect a cou- 
ple of $(u, v)$ samples from star \#1, retarget, collect a couple $(u, v)$ samples from star \#2, and then retarget back to star \#1, to collect more $(u, v)$ samples for that star. We call this approach the targeting and imaging maneuver optimization (TIMO) strategy.

Unfortunately, by allowing retarget, reorient, and resize maneuvers to be mixed, the solution space of the problem is increased considerably. Since the resulting optimization problem is NP-hard, this can present a problem. Fortunately however, our simulation results show some general patterns for how retarget, reorient, and resize maneuvers can be mixed to achieve considerable fuel savings. These patterns form the basis for the image sampling guidelines presented here.

\section{SPACE TECHNOLOGY 3}

While the methods and results of this paper are applicable to general space-based interferometry missions, to be concrete, we will develop results with respect to the planned Space Technology 3 (ST3) mission. ${ }^{6}$ This section gives a brief overview of the ST3 mission.

ST3 will be a separated spacecraft interferometer consisting of two spacecraft: a combiner and a collector, which will be launched together into an earth-trailing, heliocentric orbit. The mission will consist of three phases, a formation flying experiment mode, a short baseline monolithic interferometer mode, and a separated spacecraft observation mode. During this last mode, extending over the period of three months, the spacecraft will be separated by distances ranging from 50 meters up to one kilometer with interferometer baselines ranging from 40 to 200 meters. The combiner will combine and collect fringe data for the deconvolution of astrophysical images. The collector will act solely as a light collecting aperture. It is currently estimated that the system will be designed to image 50 stars during this period.

Optical interferometry requires the path lengths of incoming star light to be equal within fractions of the wavelength of visible light. Since only two spacecraft are available to collect light from a single source and then accurately combine the light to make fringe patterns, the spacecraft must be located such that the light path lengths are equal. Therefore the position of the spacecraft will be constrained such that the combiner is at the focus of a parabola, with the collector located on the parabola. Figure 3 shows the layout of the ST3 formation geometry. Notice the incoming starlight paths and where the spacecraft are located with respect to the reference parabola. The
Table 1: ST3 system parameters ${ }^{6}$

\begin{tabular}{ll}
\hline interferometry focus length & $10 \mathrm{~m}$ \\
maximum interferometry baseline & $200 \mathrm{~m}$ \\
minimum interferometry baseline & $40 \mathrm{~m}$ \\
mass of combiner & $350 \mathrm{~kg}$ \\
mass of collector & $250 \mathrm{~kg}$ \\
maximum thrust of combiner & $0.009 \mathrm{~N}$ \\
maximum thrust of collector & $0.009 \mathrm{~N}$ \\
specific impulse & $65 \mathrm{~s}$ \\
\hline
\end{tabular}

combiner with an internal delay line of two times the focal length is positioned at the focus of a parabola and the collector is located along the parabola to provide equal starlight path lengths. The baseline is the distance between the collector and the path of incoming starlight to the combiner.

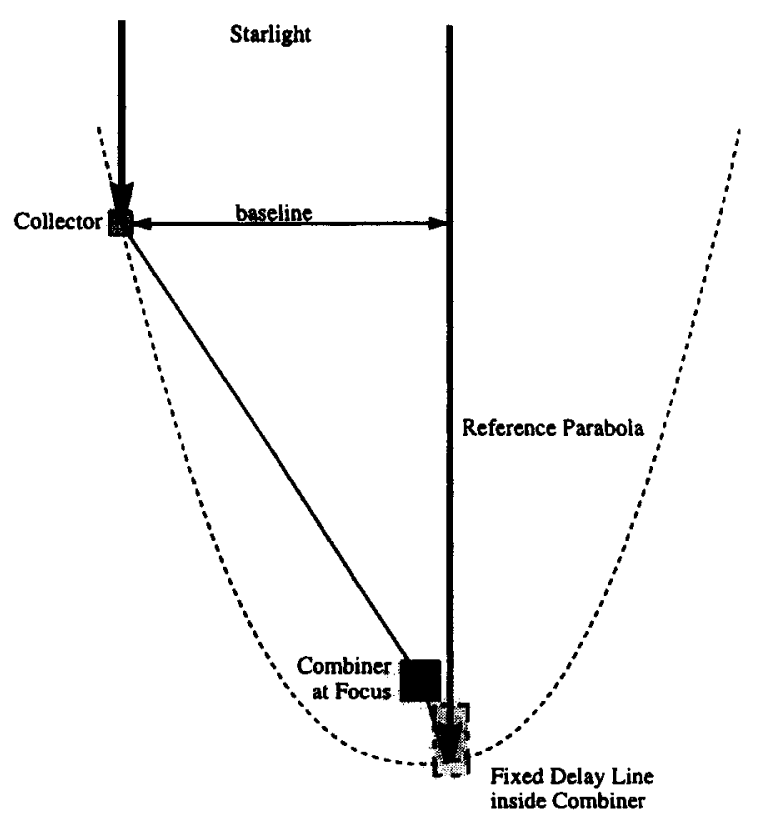

Figure 3: Layout of the ST3 two-spacecraft interferometer concept

The system parameters in Table 1 have been defined for the ST3 separated spacecraft interferometry mission. Notice that the two spacecraft have equal thrust capabilities but the combiner has more mass. 


\section{TECHNICAL APPROACH}

This section describes our approach to the the fuel optimization problem. A formation configuration corresponds to a particular relative position and orientation between the combiner and the collector, and a direction of the bore sight of the interferometer in the celestial sphere. For each star, there is a specific formation configuration required to image each $(u, v)$ point. A finite amount of fuel is required to transition between any two configurations. To compute the fuel cost between two maneuvers, we have used the results reported in Ref. 4. Alternatively, we could have used the results reported in Ref. 3 to compute the fuel costs for the case when the spacecraft are not allowed to break formation during the maneuver. Fuel costs between every possible pair of configurations were computed and tabulated in a cost matrix.

The results in Refs. 3,4 allow a tradeoff between performing fuel minimizing and fuel equalizing maneuvers for each transition. We have chosen to maneuver in such a way that fuel is equalized during each maneuver. This ensures that fuel remains equally distributed across the spacecraft throughout the planned mission.

With the cost matrix computed, the objective was to find a minimizing sequence, or tour, between the different configurations. This problem is a traveling salesman problem (TSP), which is well-known to be NP-hard. There is a tremendous amount of literature devoted to solving the TSP. Reinelt ${ }^{7}$ offers excellent insight into choosing heuristics for finding solutions, provides a good review of present and developing methods, and gives case study comparisons for chosen TSP instances. To solve the TSP problem, we have used an algorithm developed by Martin, Otto \& Felten ${ }^{8}$ called chained local optimization (CLO). The CLO algorithm combines simulated annealing with local search heuristics which have been shown to produce good results for the TSP.

\section{RESULTS}

Optical interferometry missions involve three primary types of maneuvers (retarget, resize, and reorientation) to fill out the $(u, v)$ plane for each stellar source. The targeting maneuver optimization (TMO) approach simplifies the fuel optimization problem by separating retargeting moves from imaging moves (resize and reorient) and optimizing the order of retargeting moves in which stars are imaged. In this process, only the order of retargeting moves is considered. It is assumed that all imaging moves are performed one star at a time. The (TMO) strategy results in a traveling salesman problem and is amenable to solution by discrete optimization algorithms such as CLO.

Optimizing the observation tour order can yield significant fuel-saving benefits over an ad-hoc approach. Clearly, a fuel saving strategy for an interferometry mission considering both targeting maneuvers and imaging maneuvers could result in greater reductions than considering targeting maneuvers alone. In this paper, we consider not only the targeting maneuvers for pointing the interferometer, but also all of the imaging maneuvers used to fill the $(u, v)$ plane. These fuel minimization strategies are termed targeting and imaging maneuver optimization (TIMO) strategies. Two types of imaging operations are considered with TIMO: 1-D tours involve retargeting and resizing moves, while 2-D tours involve retargeting, resizing, and reorienting moves.

\section{1-D Imaging}

Table 2 shows the different 1-D imaging scenarios. In each case three stars, separated by an angle $\beta$, are imaged. Three different values for $\beta$ are considered ( $\pi / 16, \pi / 8$, and $\pi / 4$ radians). Baseline separation distances of 10 and 20 meters are considered.

Table 2: Scheme IV 1-D test case data

\begin{tabular}{ccc}
\hline scenario & $\beta$ [radians] & baselines [meters] \\
\hline 1D1 & $\pi / 16$ & $40,50,60,70$ \\
1D2 & $\pi / 8$ & $40,50,60,70$ \\
1D3 & $\pi / 16$ & $40,60,80,100$ \\
1D4 & $\pi / 8$ & $40,60,80,100$ \\
1D5 & $\pi / 4$ & $40,60,80,100$ \\
\hline
\end{tabular}

The CLO algorithm was used to determine the tour order of pointing and resizing moves that would result in minimal fuel consumption for the 1-D test cases. The cost of the tour produced by each scenario has been compared to a benchmark tour through the same scenario. For the benchmark tour, each star is fully imaged before moving to the next star.

Results for scenarios 1D1 and 1D2 are given in Figure 4. A polar plot is used depict the results, where the three radial lines represent the three observation directions. White circles along the lines represent interferometer baseline separation distance, with the baseline increasing moving away from the origin. The left-hand plot shows the fuel usage results from the benchmark tour, while the right-hand plot shows the best CLO tour with 
its fuel consumption for each scenario. For scenario $1 \mathrm{D} 1$, the number of more costly baseline changes is minimized by the CLO algorithm. For scenario 1D2, the result is the same as the benchmark where it is better to minimize the number of retarget operations. The difference between the two scenarios is the size of the retarget angle. These results suggest that when making 10 meter baseline changes, targeting moves will be mixed with sampling moves when the retarget angle is $\pi / 16$ or less.
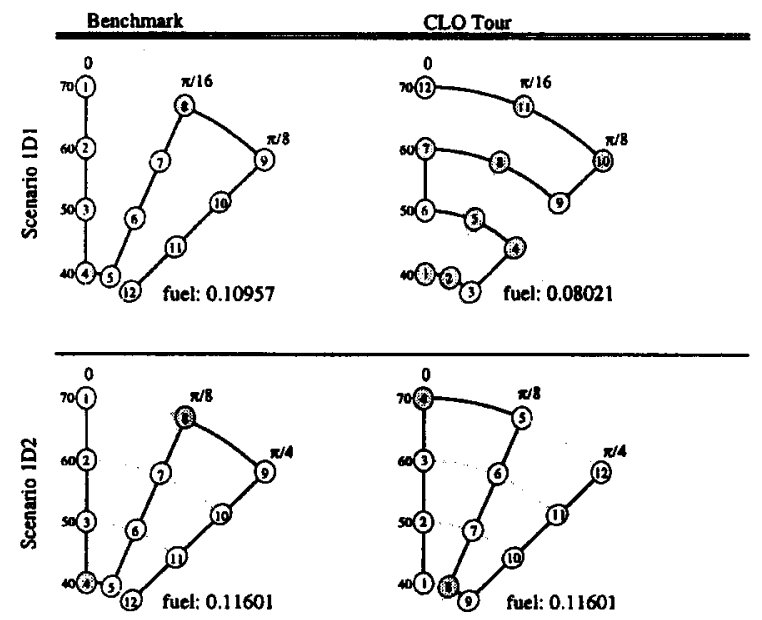

Figure 4: Tour map for scenarios 1D1 and 1D2

Results for scenarios 1D3, 1D4, and 1D5 are given in Figure 5. These scenarios require baseline changes of 20 meters. Again the difference between the scenarios is the size of the retarget angle. These results suggest that when making 20 meter baseline changes, targeting moves will be mixed with sampling moves when the retarget angle is $\pi / 8$ or less. Notice that the greatest fuel savings is realized for closely clustered observations such as the 1D3 scenario. This is expected since the pattern used in the benchmark tour is optimal for large $\beta$ angles between observation directions as can be seen in the map of scenario 1D5.

From these results, it is clear that when separation angle between stars is large, it is best to point at a star and perform all of the baseline resizing moves before moving on to the next star. Conversely, when the separation angle between stars is relatively small, less fuel is consumed when the baseline is held fixed and all of the stars are targeted before changing the baseline.

Guidelines for how these 1-D moves can be sequenced in the best way can be developed by com-
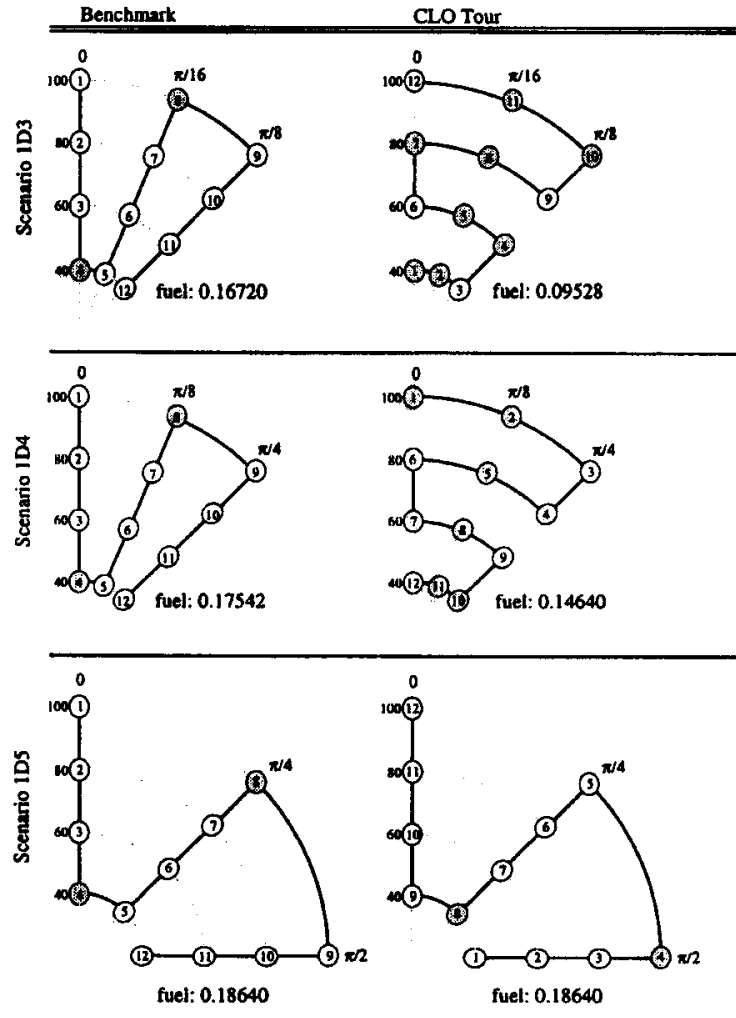

Figure 5: Tour map for scenarios 1D3, 1D4, and 1D5

paring the maximum retarget and resize costs. The maximum retarget cost, $R T_{\max }$, is defined as the cost to retarget to the next closest star when the formation is at its maximum baseline. The maximum resize cost, $R S_{\max }$, is defined as the cost to resize the formation from its maximum baseline to the next smallest baseline. Because the spacecraft are constrained to move along the reference parabola to resize the formation, the maximum resize cost will result when the spacecraft are at the largest baseline separation.

The maximum retarget and resize cost for scenarios 1D1-5 have been calculated and are shown in Table 3 with the cost inequalities. These cost inequalities suggest the priority of maneuvers to construct the fuel optimal tour.

Figure 6 shows an example of how a 1-D imaging scenario might be constructed using only the maximum retarget and resize costs $\left(R T_{\max }\right.$ and $\left.R S_{\max }\right)$ as a guide. The numbers inside of the circles represent the tour order. Gray shaded circles indicate that the next maneuver is a retarget. By comparing, $R T_{\max }$ and $R S_{\max }$, a priority ranking can be 
Table 3: 1-D test case maximum retarget and resize costs

\begin{tabular}{cccc}
\hline scenario & $R T_{\max }$ & $R S_{\max }$ & priority \\
\hline 1D1 & 0.0099 & 0.0112 & $R S_{\max }>R T_{\max }$ \\
1D2 & 0.0139 & 0.0112 & $R T_{\max }>R S_{\max }$ \\
1D3 & 0.0138 & 0.0185 & $R S_{\max }>R T_{\max }$ \\
1D4 & 0.0194 & 0.0185 & $R T_{\max }>R S_{\max }$ \\
1D5 & 0.0272 & 0.0185 & $R T_{\max }>R S_{\max }$ \\
\hline
\end{tabular}

given to the lower cost maneuver. When it is more costly to retarget than resize $\left(R T_{\max }>R S_{\operatorname{mex}}\right)$, as shown in illustration 1 of this figure, a fuel minimal tour should resize until it must retarget. In this case, a fuel optimal tour results when the number of more costly moves (retargets) is minimized. There must be at least two retargets to complete the mission. Alternatively, when resizing is more expensive than retargeting $\left(R S_{\max }>R T_{\max }\right)$ as shown in illustration 2, the tour should retarget to each star in the cluster before changing baselines. A minimum of three resize maneuvers are required as shown in illustration 2 of Figure 6.

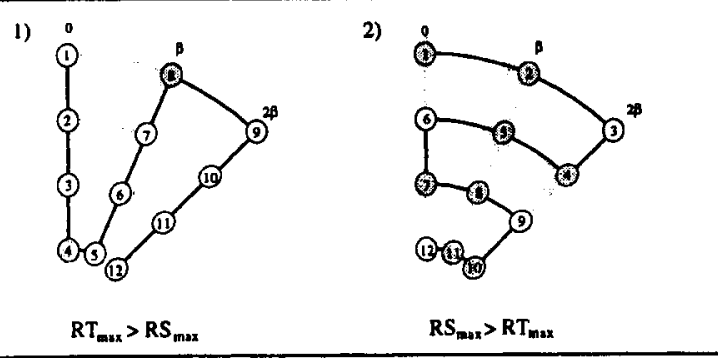

Figure 6: Sampling pattern guide for 1-D imaging

When the sampling pattern guidelines are applied to the five 1-D test cases, the results are identical to those obtained by the CLO algorithm with the exception of case 1D4. The guide tour from scenario $1 \mathrm{D} 4$ was more costly than the tour found with CLO. From Table 3 it can be seen that for scenario 1D4, $R T_{\max }$ is only slightly larger than $R S_{\max }$. Thus, the sampling guidelines suggest that the number of baseline resize moves should be minimized. Because the guidelines only consider the maximum retarget or resize cost and not the total cost of all of the possible retarget or resize moves, it is possible that when $R T_{\max }$ and $R S_{\max }$ are close in value, the guidelines may not lead to the optimal fuel savings. It should be noted that under these conditions, the CLO tour and guideline tour fuel usage differ only slightly.

\section{2-D Imaging}

The 2-D test case scenarios were designed to investigate fuel saving strategies when retargeting, reorienting, and resizing moves are involved. The retargeting angle between stars for each test case scenario investigated is shown in Table 4. For each scenario, the baseline distances are 40, 50 and 60 meters, with orientations about the observation direction of $0, \pi / 8, \pi / 4,3 \pi / 8$, and $\pi / 2$ radians for each scenario.

Table 4: Scheme IV 2-D test case data

\begin{tabular}{cc}
\hline scenario & $\beta$ [radians] \\
\hline $2 \mathrm{D} 1$ & $\pi / 8$ \\
$2 \mathrm{D} 2$ & $\pi / 4$ \\
$2 \mathrm{D} 3$ & $3 \pi / 8$ \\
$2 \mathrm{D} 4$ & $\pi / 2$ \\
\hline
\end{tabular}

The CLO algorithm was used to find tours for each of these four scenarios. Following the run of each scenario, the mission was simulated using the CLO-suggested tour to determine the amount of fuel expended. Figures 7 and 8 show CLO results for scenarios 2D1 and 2D2. In each figure, the resultant CLO tour and its fuel consumption is shown with a benchmark tour and its fuel consumption for comparison. These figures use a polar plot similar to those used for the 1-D tours, however the representation is different. Here, two plots are given, where each plot represents an observation in the directions shown by the bold arrows in the upper left corner of the figure. The radial lines in each plot represent the orientation of the spacecraft pair about the different observation directions. The white circles along the radial lines represent the baseline separation distance as before. For the benchmark tour, each star is fully imaged before moving to the next star, with baseline resize moves being carried out at each orientation before moving to the next orientation. The mission durations for the CLO tours are adjusted to take the same amount of time as the benchmark tour to execute the maneuvers required to perform the mission objectives.

Scenario 2D1 shown in Figure 7 images two stars separated by a $\beta$ angle of $\pi / 8$. The CLO tour samples at all orientations with a single baseline for both stars before moving to the next baseline. Note the fuel savings realized by the CLO tour. The figure demonstrates that for stars closely clustered together, mixing targeting and sampling maneuvers 


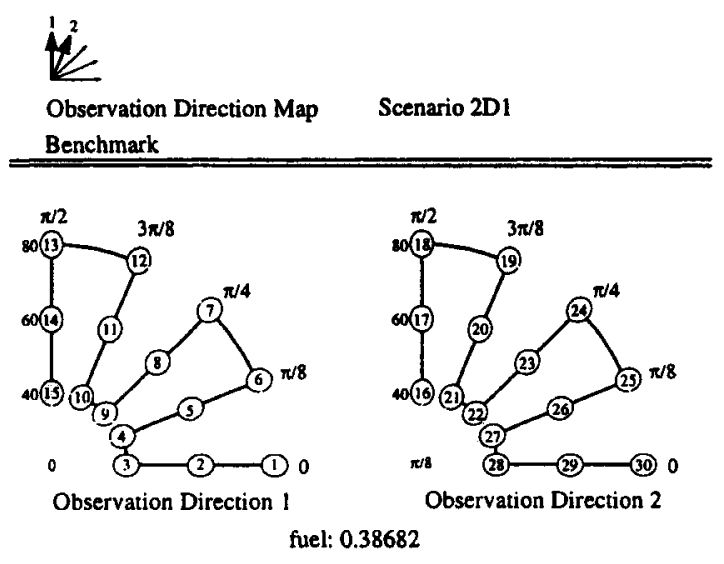

CLOTour

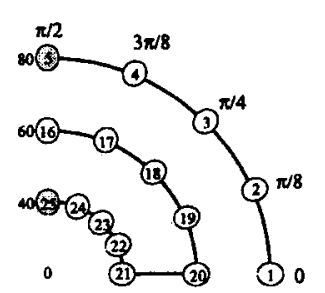

Observation Direction 1 fuel: 0.20925

Figure 7: Tour map for scenarios 2D1

provides a large reduction in fuel consumption compared to the benchmark tour.

The 2D2 scenario shown in Figure 8 images two stars separated by a $\beta$ angle of $\pi / 4$. Like the baseline tour, the CLO tour also entirely samples one star but samples at all orientations of that star with a single baseline before changing to the next baseline. Note the fuel savings by the CLO tour. Even though each star is imaged separately, the CLO tour is still more fuel efficient.

The maximum retarget, reorient, and resize cost for scenarios 2D1 through 2D4 have been calculated and are shown in Table 5 with the priority cost inequalities. These cost inequalities suggest the priority of maneuvers to construct the fuel optimal tour. $R T_{\max }$ and $R S_{\max }$ are calculated the same way as for $1-\mathrm{D}$ imaging. The maximum reorient cost, $R O_{\text {max }}$, is defined as the cost to reorient to the next closest orientation when the formation is at its maximum baseline. This will be the maximum reorient cost since the spacecraft must travel further to reorient at larger baselines.

By examining the priority cost inequalities for sce-

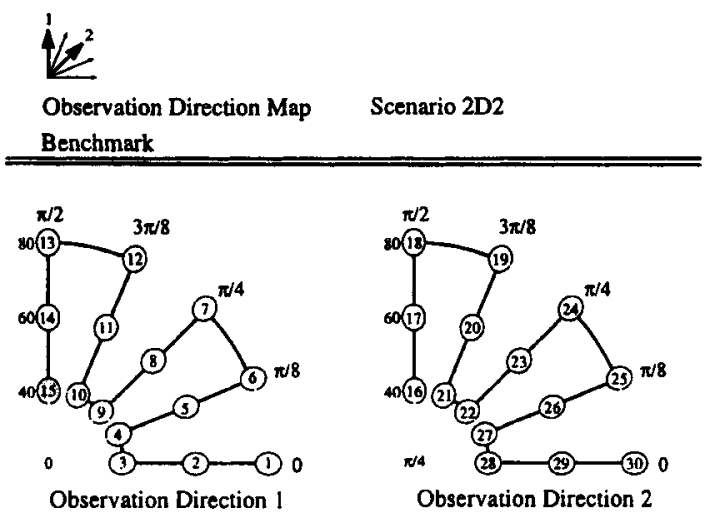

fuel: 0.38945

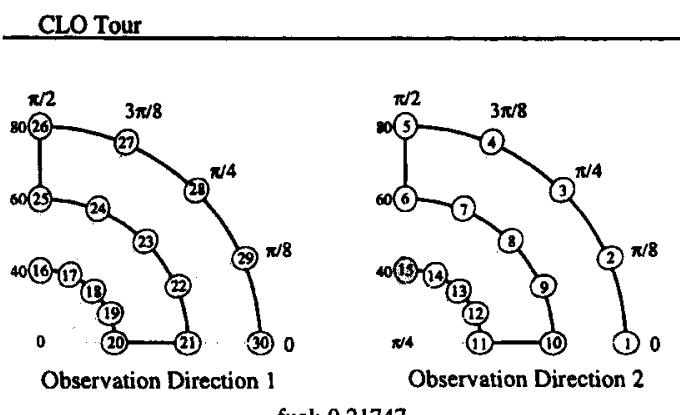

Figure 8: Tour map for scenarios 2D2

narios 2D2, 2D3, and 2D4, it is expected that the optimal sampling patterns for scenarios 2D3 and 2D4 would be the same as optimal pattern for scenario 2D2. This has been confirmed by CLO results for these scenarios.

Table 5: 2-D maximum retarget, reorient, and resize costs

\begin{tabular}{ccccc}
\hline scen. & $R T_{\max } R O_{\max }$ & $R S_{\max }$ & priority \\
\hline 2D1 & 0.0158 & 0.0108 & 0.0164 & $R S_{\max }>R T_{\max }>R O_{\max }$ \\
2D2 & 0.0221 & 0.0108 & 0.0164 & $R T_{\max }>R S_{\max }>R O_{\max }$ \\
$2 \mathrm{D}$ & 0.0266 & 0.0108 & 0.0164 & $R T_{\max }>R S_{\max }>R O_{\max }$ \\
$2 \mathrm{D} 4$ & 0.0300 & 0.0108 & 0.0164 & $R T_{\max }>R S_{\max }>R O_{\max }$ \\
\hline
\end{tabular}

Using these priority cost inequalities, guideline tours can be constructed. For example, in case 2D1 reorient moves use the least amount of fuel. Accordingly, the tour remains on the same target and baseline until no further reorient moves can be made (tour stop 5 of Figure 7). At this point, the priority suggests that it is cheaper to retarget than to resize,

7

American Institute of Aeronautics and Astronautics 
so a retarget move is made from stop 5 to stop 6 . The tour then reorients from stop 6 through stop 10 , where the first baseline change is made. Since $R S_{\max }$ has the lowest priority for this scenario, the objective is to make the minimum number of resize moves while completing the mission requirements.

Using such reasoning, guideline tours can be developed for every possible cost priority. The guideline tours in Figure 9 result in the same sampling patterns as the CLO algorithm for scenarios 2D1 through 2D4. The guideline tours address all of the possible scenarios for 2-D imaging of multiple stars and provide systematic methods for sweeping out the sampling pattern in a fuel efficient way. The 2-D guide tours are not the result of a formal optimization process, however, for all of the test cases considered they gave results identical to those from the CLO algorithm.

Illustrations 3 through 8 of Figure 9 show how a 2-D observation sequence might be constructed by comparing the maximum retarget, reorient, and resize costs. Scenario 2D1 discussed above corresponds to illustration 5 and scenario 2D2 corresponds to illustration 4. Guide tours for each of the other combinations of maximum repositioning costs, $R T_{\max }, R O_{\max }$, and $R S_{\max }$ are determined following the same priority path system. For example, in illustration 8 , since $R O_{\max }>R S_{\max }>R T_{\max }$, the number of reorient moves should be minimized followed by the number of resize moves. The minimum number of reorient moves is four and the minimum number of resize moves is ten. Clearly, Figure 9 can help determine how the sampling pattern should be mapped out to fill the $(u, v)$ plane in the most fuel efficient way. The guidelines can be used to form final sampling plan for a mission or as an initial condition for further optimization studies. Table 6 summarizes the minimum number of basic maneuvers that must be performed for each of the six priority paths to construct fuel minimal tours. Since there are 30 tour stops, and only basic maneuvers can be used to travel between stops, 29 maneuvers must be performed. Therefore, the sum of all retarget, reorient, and resize maneuvers will be 29 . Notice in this table that the number of basic maneuvers for each illustration, matches the priority inequality.

For similar baseline-change sampling, the results of the 2-D scenarios are consistent with the results from the 1-D scenarios. Notice that the CLO algorithm chose to mix targeting and sampling maneuvers when the retargeting $\beta$ angle was $\pi / 8$ as in scenarios 1D4 and 2D1. For $\beta$ angles of $\pi / 4$, the tour images each star separately as shown by scenarios $1 \mathrm{D} 5$ and 2D2.
3)
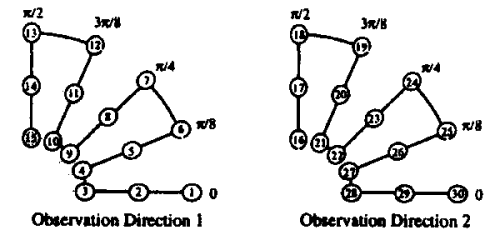

$$
\mathrm{RT}_{\max }>\mathrm{RO}_{\max }>\mathrm{RS}_{\max }
$$

4)

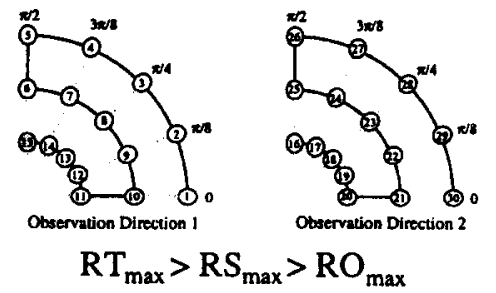

5)
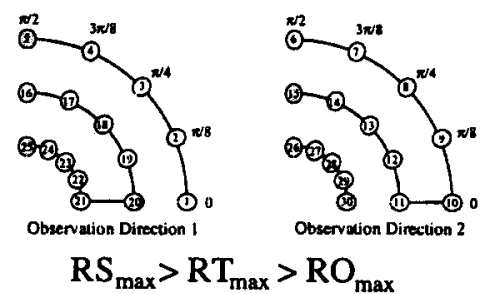

6)

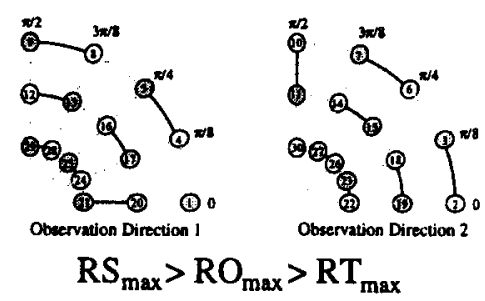

7)
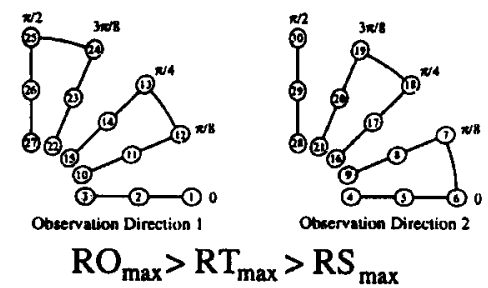

8)

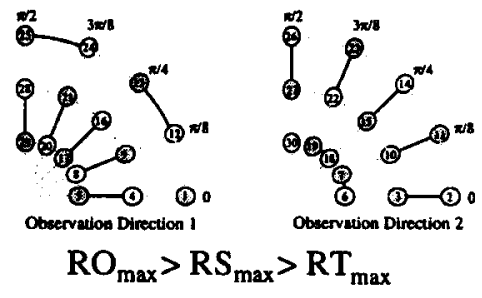

Figure 9: Sampling pattern guide for 2-D imaging 
Table 6: 2-D priority guide to the minimal number of basic maneuvers for fuel minimal tours

\begin{tabular}{cccc}
\hline illustration & retarget & reorient & resize \\
\hline 3 & 1 & 8 & 20 \\
4 & 1 & 24 & 4 \\
5 & 3 & 24 & 2 \\
6 & 15 & 12 & 2 \\
7 & 5 & 4 & 20 \\
8 & 15 & 4 & 10 \\
\hline
\end{tabular}

\section{Fuel Savings}

Table 7 shows the percentage of savings of the guide tour below the benchmark tour for each of the TIMO scenarios tested. Where the percent savings is 0.0 , the benchmark tour is the same as the guide tour. Mixing of targeting and sampling moves took place

Table 7: Percent Savings of TIMO test cases below benchmark

\begin{tabular}{cc}
\hline scenario & percent savings \\
\hline 1D1 & 36.6 \\
1D2 & 0.0 \\
1D3 & 75.5 \\
1D4 & 0.0 \\
1D5 & 0.0 \\
2D1 & 84.9 \\
2D2 & 79.1 \\
2D3 & 76.7 \\
2D4 & 75.7 \\
\hline
\end{tabular}

on scenario $2 \mathrm{D} 1$ where the savings was the greatest. Notice that even when no mixing occurs in scenarios (e.g., 2D2, 2D3, and 2D4), significant savings below the benchmark tour are still realized. This is accomplished by optimizing the order of reorient and resize moves. This suggests that even if the stars are not tightly clustered and only one star is imaged at a time, the sampling pattern is still an important consideration for fuel efficiency.

\section{CONCLUSIONS}

Significant fuel savings can be realized by combining retargeting moves used to point the formation towards the stellar source with imaging moves used to position the formation about the observation direction to take the multiple samples necessary for optical interferometry. As examples of the potential fuel savings that can be realized, fuel optimal tours were compared with a benchmark tour for imaging two stars. Simulation results showed fuel savings up to $85 \%$ lower than the the benchmark tour. The fuel savings were most significant for test cases involving closely clustered stars. For stars with large separation angles, targeting moves are carried out independent of the imaging moves. Even so, significant fuel savings can be achieved by considering the sequence of the imaging maneuvers alone.

\section{REFERENCES}

[1] M. Shao and M. M. Colavita, "Long-baseline optical and infrared stellar interferometry," Annual Review of Astronomy and Astrophysics, vol. 30, pp. 457-498, 1992.

[2] S. S. Joshi, "An informal introduction to synthetic aperture imaging," Interoffice Memorandum 3450-98-0004, Jet Propulsion Laboratory, 4800 Oak Grove Dr., Pasadena, CA 91109, February 1998.

[3] R. W. Beard, T. W. McLain, and F. Y. Hadaegh, "Fuel optimization for constrained rotation of spacecraft formations," AIAA Journal of Guidance, Control, and Dynamics, vol. 23, pp. 1-8, January-February 2000.

[4] R. W. Beard and F. Y. Hadaegh, "Fuel optimized rotation for satellite formations in free space," in American Control Conference, (San Diego, CA), 1999.

[5] C. A. Bailey, "Fuel saving schemes for separated spacecraft flying in formation," Master's thesis, Brigham Young University, Department of Mechanical Engineering, December 1999.

[6] JPL, "Exhibit 1: Deep Space 3 spacecraft system requirements." Exhibit 1 of Request for Proposal RFP NO. N01-4-9048-213 for Deep Space 3 (DS3) Spacecraft System Industry Partner.

[7] G. Reinelt, The Traveling Salesman: Computational Solutions for TSP Applications. SpringerVerlag, 1994.

[8] O. C. Martin, S. W. Otto, and E. W. Felten, "Large-step markov chains for the traveling salesman problem," Complex Systems, vol. 5, no. 3, p. 299, 1991. 